\title{
3-Deoxy-D-manno-2-octulosonic acid in the lipopolysaccharide of various strains of Pseudomonas cepacia
}

\author{
D. C. STRAUS, MIRIAM K. LONON, D. E. WOODSt and C. W. GARNER*
}

Department of Microbiology and ${ }^{\bullet}$ Department of Biochemistry and Molecular Biology, Texas Tech University Health Sciences Center, Lubbock, Texas 79430, USA, and TDepartment of Microbiology and Infectious Diseases, The University of Calgary Health Sciences Centre, Calgary, Alberta, Canada T2N 4N1

\begin{abstract}
Summary. Six clinical isolates of Pseudomonas cepacia (representing the five serotypes of the organism) were examined for the presence of 3-deoxy-D-manno-2-octulosonic acid (KDO) in their lipopolysaccharide (LPS). Purified LPS was examined for the presence of KDO by the thiobarbituric acid (TBA) assay and by gas chromatography. All strains possessed KDO. One strain possessed KDO that was detectable by the TBA assay after mild acid hydrolysis with $0.04 \mathrm{M} \mathrm{H}_{2} \mathrm{SO}_{4}$ at $100^{\circ} \mathrm{C}$ for $20 \mathrm{~min}$. The other strains also possessed KDO but it was only demonstrable by the TBA assay after strong acid hydrolysis $\left(4 \mathrm{M} \mathrm{HCl}\right.$ for $60 \mathrm{~min}$ at $100^{\circ} \mathrm{C}$ ). All six purified LPS preparations were shown to possess $\mathrm{KDO}$ by two separate gas chromatography procedures. LPS isolated from the six strains of $P$. cepacia was toxic for mice.
\end{abstract}

\section{Introduction}

The consequences of Pseudomonas cepacia infection in cystic fibrosis (CF) patients are receiving much attention, ${ }^{1}$ because $\mathrm{CF}$ patients are currently the largest group of individuals infected with this bacterium. $^{2-4}$

Because so little is known about the virulence factors of $P$. cepacia, we decided to examine one aspect of a virulence factor about which controversy presently exists - the presence or absence of 3deoxy-D-manno-2-octulosonic acid (KDO) in the lipopolysaccharide (LPS). Maniello et al.$^{5}$ were the first to state that they could find no detectable KDO in LPS extracted from two strains of $P$. cepacia when they employed the procedure of Osborn. ${ }^{6}$ Anwar et $a{ }^{7}{ }^{7}$ were also unable to detect any KDO in the outer membrane of one strain of $P$. cepacia. However, Straus et al. ${ }^{8}$ reported the isolation of KDO from the culture supernate of 2 of 10 strains of $P$. cepacia they examined. Their material gave a positive thiobarbituric acid (TBA) reaction product, ${ }^{6}$ which absorbed strongly at $549 \mathrm{~nm}$, and appeared to be spectrally identical to that produced by the purified KDO used as the standard. In the present study we examined the LPS of six clinical isolates of $P$. cepacia for the presence of $\mathrm{KDO}$ by TBA assay and by gas chromatography.

Received 13 March 1990; accepted 24 May 1990.

\section{Materials and methods}

\section{Bacteria and media}

The six strains of $P$. cepacia employed in this study were isolated from sputum samples from patients with $\mathrm{CF}$; their origins and serotypes were as follows: strains Pc61g (serotype D), Pc527i (D) and Pc99bb (B) from Dr J. D. Klinger of Integrated Genetics, Framington, MA; strains K63-2 (E) and K19-2 (A) from Dr C. L. Prober, The Hospital for Sick Children, Toronto, Ontario; and strain 5530pk (C) was isolated at Alberta Children's Hospital, Calgary, Alberta, Canada. The serotyping of these strains has been described ; ${ }^{9}$ storage and handling of the organisms were as previously described. ${ }^{10}$

\section{Determination of KDO in purified LPS}

Cultures were grown in $10 \mathrm{~L}$ of $\mathrm{M}-9$ medium $^{11}$ containing glucose $0.2 \%$ with shaking (180 revolutions per min) at $37^{\circ} \mathrm{C}$ for $24 \mathrm{~h}$. The samples were immediately chilled on ice and centrifuged at $17700 \mathrm{~g}$ for $30 \mathrm{~min}$ at $4^{\circ} \mathrm{C}$. LPS was then extracted by the procedure of Darveau and Hancock. ${ }^{12}$ Purified LPS preparations were examined for protein content by the procedure of Lowry $e t$ $a l{ }^{13}$ and for nucleic acid content by the method of Kabat and Mayer. ${ }^{14}$

Purified LPS preparations were assayed for KDO by the procedure described by Osborn ${ }^{6}$ with KDO (Sigma) as the standard. This assay with a $0.04 \mathrm{M} \mathrm{H}_{2} \mathrm{SO}_{4}$ hydrolysis step for $20 \mathrm{~min}$ at $100^{\circ} \mathrm{C}$ was considered to be mild hydrolysis; strong acid hydrolysis involved exposure to 
$4 \mathrm{M} \mathrm{HCl}$ at $100^{\circ} \mathrm{C}$ for $60 \mathrm{~min}$. The presence of $\mathrm{KDO}$ in these hydrolysed preparations was determined by spectral analysis in the range $500-600 \mathrm{~nm}$ in a Beckman DU-70 spectrophotometer. Samples from which a curve similar to that of purified KDO was obtained, with an optimum absorbance at $550 \mathrm{~nm}$, were considered probably to contain KDO.

KDO in purified LPS was analysed by gas chromatography on two different columns as the methyl glycoside trifluoroacetate as follows. Purified LPS preparations (5$10 \mathrm{mg}$ ) were dried under a stream of nitrogen at $40-50^{\circ} \mathrm{C}$ and kept under vacuum overnight. Methanolysis was accomplished by heating each sample in $2.0 \mathrm{ml}$ of $1.5 \mathrm{M}$ $\mathrm{HCl}$ in dry methanol (Supelco) at $80^{\circ} \mathrm{C}$ for $18 \mathrm{~h}$ under nitrogen. The methanol was removed under a stream of nitrogen at $40^{\circ} \mathrm{C}$. Methanol was added and evaporation was repeated once to remove residual $\mathrm{HCl}$. The samples were then kept under vacuum for at least $1 \mathrm{~h}$. Acetonitrile $(200 \mu \mathrm{l})$ and then trifluoroacetic anhydride $(200 \mu \mathrm{l})$ were added and the samples were heated to $80^{\circ} \mathrm{C}$ for $30 \mathrm{~min}$. The volume of each sample was reduced to $c$. $50 \mu \mathrm{l}$ under a stream of nitrogen at $25^{\circ} \mathrm{C}$. Samples were chromatographed on a $12-\mathrm{m}$ capillary column, with an internal diameter of $0.32 \mu \mathrm{m}$ coated with SE-30 (Supelco) in a Varian Model 3300 Gas Chromatograph equipped with a flame-ionisation detector. The carrier gas was nitrogen with stream-splitting at 1 in 100 . The initial temperature was held at $70^{\circ} \mathrm{C}$ for $1 \mathrm{~min}$ and then increased to $150^{\circ} \mathrm{C}$ at $4^{\circ} \mathrm{C} / \mathrm{min}$. KDO (Sigma) was used as a standard. In the second procedure, the methyl glycoside trifluoroacetates were chromatographed on a $15-\mathrm{m}$ capillary column with an internal diameter of $0.32 \mu \mathrm{m}$, coated with SP-2250 (Supelco) at an initial temperature of $100^{\circ} \mathrm{C}$. The temperature was raised to $160^{\circ} \mathrm{C}$ at $4^{\circ} / \mathrm{min}$.

The LPS preparations from each of the six strains were examined for their toxicity in experimental animals. The LD50 values of the purified, UV sterilised LPS preparations were determined by intraperitoneal (i.p) injection into Swiss-Webster mice. After $72 \mathrm{~h}$, the number of dead mice in each group was determined and LD50 values were calculated by the method of Reed and Muench. ${ }^{15}$

\section{Results}

\section{Occurrence of $K D O$ in P. cepacia}

Strain Pc61g, serotype D, contained KDO in a form that could be detected by the TBA assay after mild acid hydrolysis ${ }^{6}$ (fig. 1). Purified LPS of strain Pc61g gave a distinctly positive TBA reaction, which absorbed maximally at $550 \mathrm{~nm}$ and was spectrally identical to the purified KDO used as a standard. With the other five strains, material giving this absorbance was detected only after strong hydrolysis which did, however, destroy much of the KDO.

Proof that the purified LPS from strain Pc99bb possessed KDO was afforded by gas chromatogra-
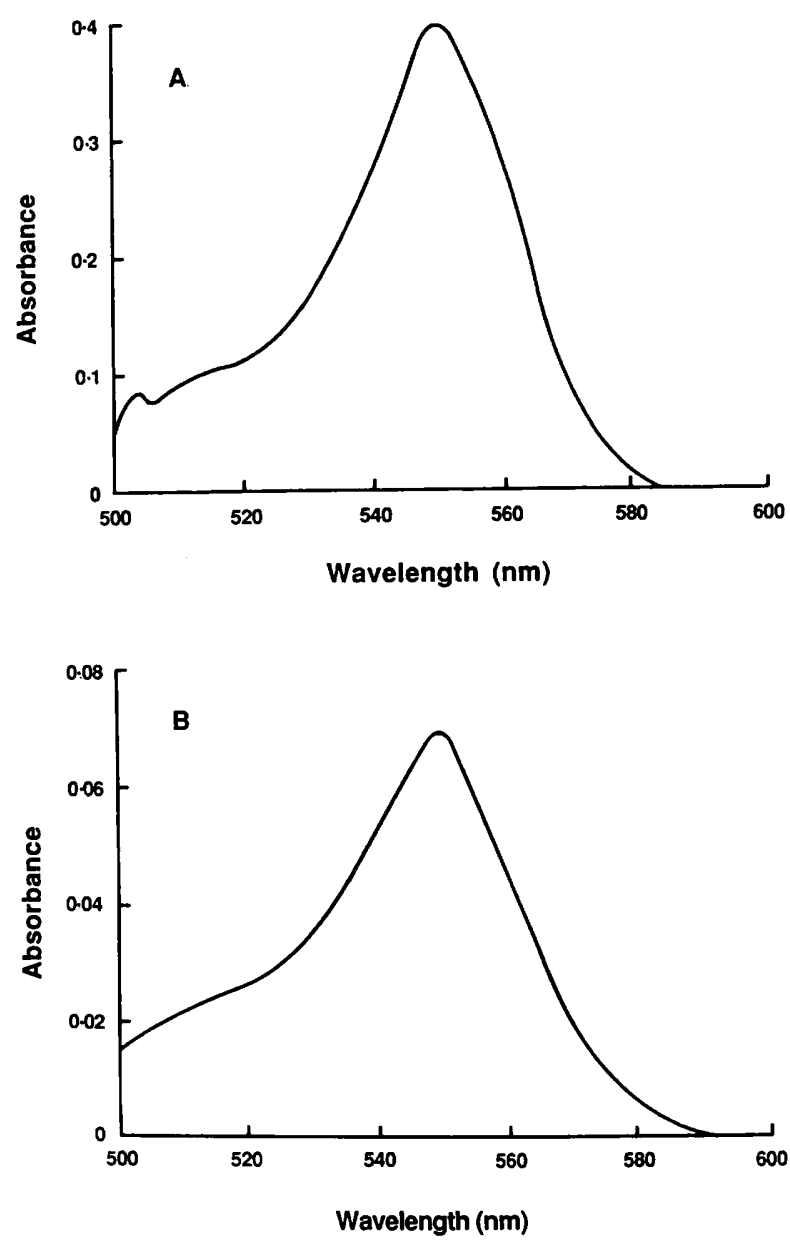

Fig. 1. Absorption spectrum of (A) $5.0 \mu \mathrm{g}$ of KDO standard and (B) $50 \mu \mathrm{l}$ of purified LPS from strain Pc6lg.

phy. Purified KDO in SE-30 gas chromatography gave a retention profile with four peaks at approximately $13.2 \mathrm{~min}$ (minor), 14.4 min (minor), 16.3 $\mathrm{min}$ and $17.0 \mathrm{~min}$ (data not shown). These peaks at $16.3 \mathrm{~min}$ and $17.0 \mathrm{~min}$ were seen in the profile of the purified LPS from strain Pc99bb (fig. 2) as well as in the SE-30 profiles of the other five purified LPS preparations (data not shown). The peaks at $13.2 \mathrm{~min}$ and $14.4 \mathrm{~min}$ occur in regions of the chromatogram containing numerous peaks, which makes their use in identification of KDO questionable.

Fig. 3 shows the retention times of KDO in gas chromatography on SP-2250 columns. Again, four peaks were observed in standards with retention times as $7.1 \mathrm{~min}$ (minor), $8.4 \mathrm{~min}$ (minor), $9.4 \mathrm{~min}$ and $12.3 \mathrm{~min}$. The peaks at 7.1 and $8.4 \mathrm{~min}$ were not useful for KDO analysis. On the basis of 


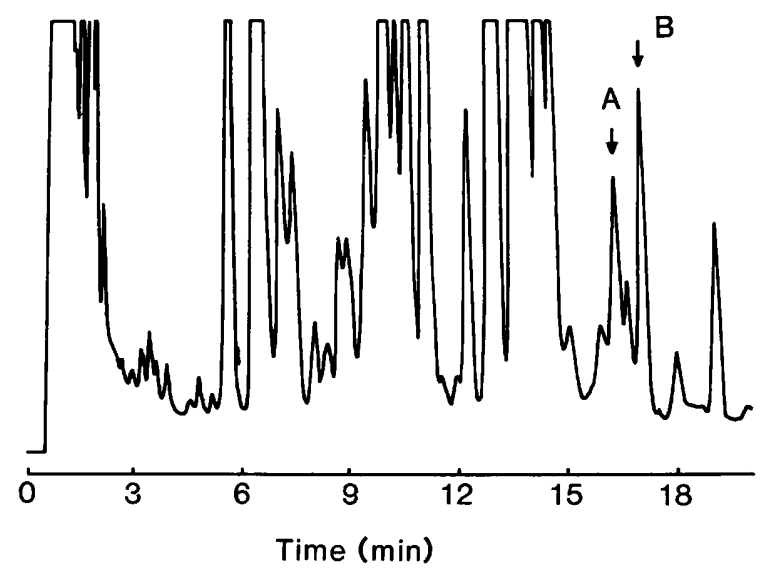

Fig. 2. Retention time profile of O-methyl glycoside trifluoroacetates prepared from the purified LPS from strain Pc99bb by gas chromatography with SE-30 as the immobile phase. The arrows indicate the two KDO peaks (A and B) that were found in all LPS samples.

differences in peak areas, we believe that the peaks at 9.4 and $12.3 \mathrm{~min}$ (SP-2250) are derived from the same substances that give rise to peaks at 17.0 and 16.3 min respectively on SE-30 chromatography. These peaks are labelled " $A$ " and " $B$ " in figs 2 and 3 to indicate this. The presence of both peaks is indicative of KDO. Multiple peaks are believed to result from $\alpha$ and $\beta$ anomers of pyranoside and furanoside ring structures of KDO methyl glycoside trifluoroacetates.

Fig. 4 shows the absorption spectrum of TBAreactive material in purified LPS from strain Pc99bb after mild and strong acid hydrolysis. LPS from strain Pc99bb produced a chromogen with a

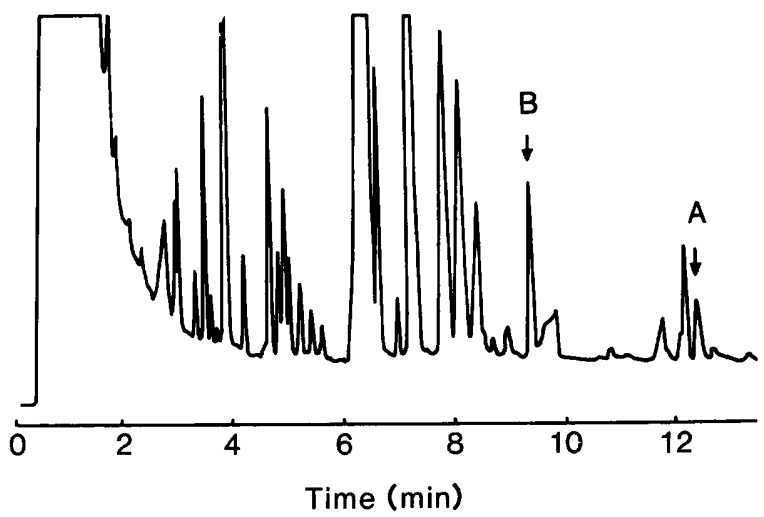

Fig. 3. Retention time profile of O-methyl glycoside trifluoroacetates prepared from the purified LPS from strain Pc99bb by gas chromatography with SP-2250 as the immobile phase. Peaks $A$ and $B$ in the profile are believed to be identical to peaks A and $B$, respectively, in the profile in fig. 2 . maximum absorbance at $536 \mathrm{~nm}$, which is not indicative of $\mathrm{KDO}$ (fig. 4A); however, after strong acid hydrolysis (fig. 4B), the same material produced a TBA-reactive compound with a maximum absorbance at $550 \mathrm{~nm}$ typical of KDO.

\section{Characterisation and toxicity of LPS preparations from $P$. cepacia}

All six LPS preparations contained $<0.6 \%$ protein and $<1 \%$ nucleic acid. The table shows the absorbance maxima of purified LPS obtained from the six $P$. cepacia isolates after strong hydrolysis and the LD50 values of these substances for mice. All six LPS preparations were more or less equally toxic for mice when injected i.p. There was no
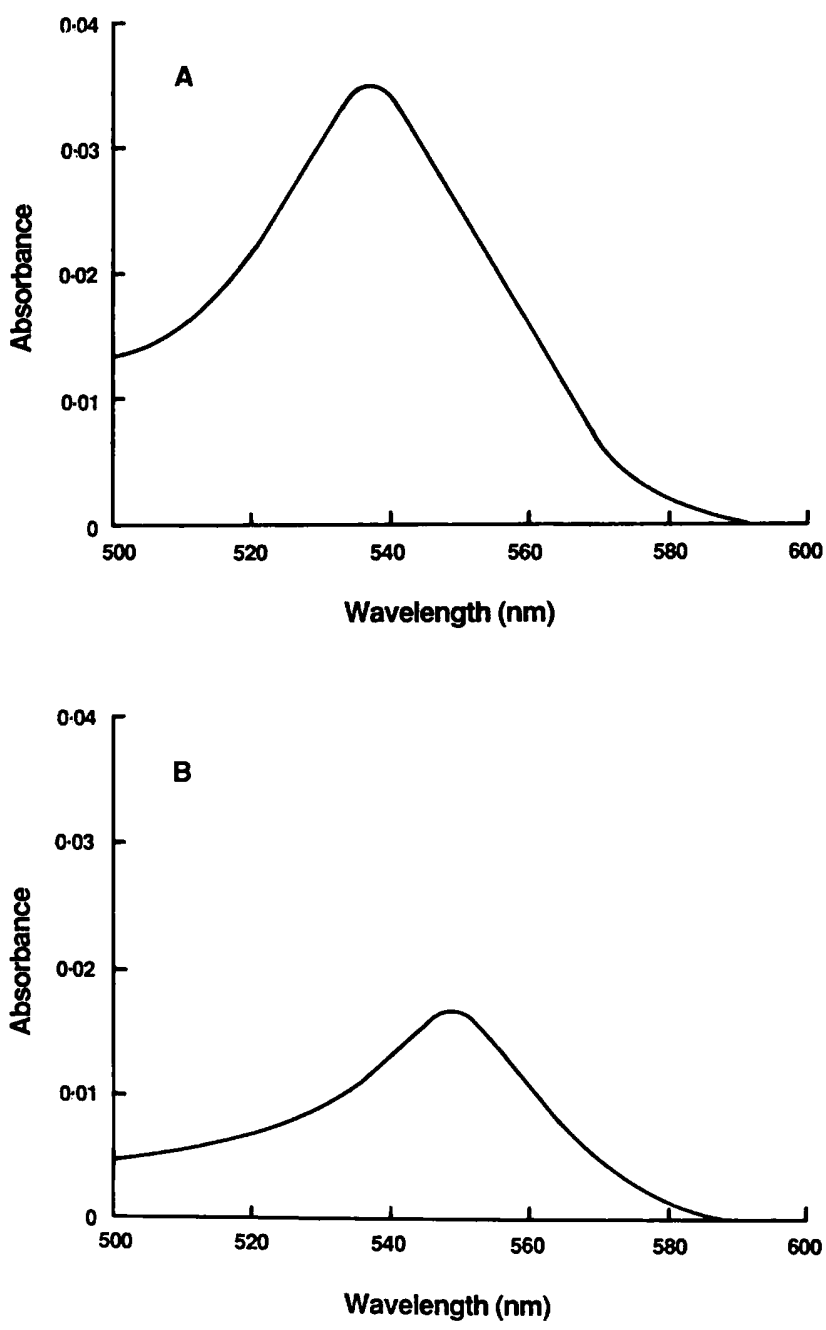

Fig. 4. Visible absorption spectrum of $50 \mu \mathrm{l}$ of purified LPS from $P$. cepacia strain $99 \mathrm{bb}$ after (A) mild and (B) strong acid hydrolysis followed by the thiobarbituric acid assay. 
Table. KDO determinations and LD50 values in mice of purified LPS from clinical isolates of $P$. cepacia

\begin{tabular}{|c|c|c|c|}
\hline Strain & Serotype & $\begin{array}{l}\text { LPS } \\
\text { LD50 } \\
(\mu \mathrm{g})\end{array}$ & $\begin{array}{l}\text { Absorbance maxi- } \\
\text { mum after strong } \\
\text { hydrolysis }\end{array}$ \\
\hline K19-2 & A & 1000 & 550 \\
\hline Pc99bb & B & 1500 & 550 \\
\hline 5530pk & C & 1250 & 550 \\
\hline Pc527i & D & 1100 & 550 \\
\hline Pc61g & D & 1000 & 550 \\
\hline K63-2 & E & 1250 & 550 \\
\hline
\end{tabular}

correlation between the toxicity of the preparation and the severity of the hydrolysis conditions required for the detection of KDO.

\section{Discussion}

The detection of KDO in gram-negative bacterial LPS is usually achieved by the TBA assay..$^{6-8,10}$ In this assay, KDO reacts as a free unsubstituted monosaccharide, and thus has to be liberated from LPS by hydrolysis.

KDO-deficient endotoxins have been reported in several bacterial species. ${ }^{16}$ This conclusion is usually based upon the lack of reactivity of LPS in the TBA assay. $P$. cepacia is one of the gramnegative organisms whose LPS was originally reported not to contain KDO. ${ }^{5}$ This finding was confirmed by Anwar et al. ${ }^{7}$ in their examination of the outer membrane of one strain of $P$. cepacia. However, Straus et al. ${ }^{10}$ reported that they could detect extracellular KDO in approximately $20 \%$ of the $P$. cepacia strains they examined. In another study, Straus et al. ${ }^{8}$ confirmed the presence of KDO in approximately $20 \%$ of the $P$. cepacia strains; sweet potato acid phosphatase had no effect on the amount of KDO detected.

By employing capillary gas chromatography, we have now demonstrated that KDO is present in the purified LPS of all six strains of $\boldsymbol{P}$. cepacia examined. It appears that approximately $80 \%$ of $P$. cepacia strains produce an LPS that does not react with the TBA reagent after mild acid hydrolysis. For KDO to be reactive in the TBA assay, the - $\mathrm{OH}$ groups in the $\mathrm{C}-4$ and $\mathrm{C}-5$ positions must be unsubstituted ; however, if the $-\mathrm{OH}$ group in the $\mathrm{C}-5$ position is substituted, the $C-4, C-6$ and $C-7$ positions must be free. ${ }^{17}$ The majority of $P$. cepacia strains contain
KDO that is detectable only after strong acid hydrolysis which indicates some form of substitution in one of the appropriate carbon positions.

The purified LPS from strain Pc61g after mild acid hydrolysis gives a maximum absorbance at $550 \mathrm{~nm}$. This indicates that this strain possesses $\mathrm{KDO}$ that is unsubstituted in one of the carbon positions that would block this reactivity. Gas chromatography on SE-30 demonstrated KDO in our six LPS preparations. Purified KDO yielded characteristic peaks at 16.3 and $17.0 \mathrm{~min}$; these peaks were seen in chromatograms of all six LPS preparations. Chromatography on a SP-2250 column confirmed the presence of KDO.

Endotoxins lacking KDO have been described in Bacteroides spp. ${ }^{18}$ Pseudomonas spp. ${ }^{19}$ and Aeromonas spp. ${ }^{20}$ These observations were usually based on the absence of TBA-reactive material after mild acid hydrolysis which liberates the fragment $\left(\mathrm{OHC}-\mathrm{CH}_{2}-\mathrm{CO}-\mathrm{COOH}\right)$; reactive material cannot be thus generated if $\mathrm{KDO}$ is substituted at certain carbon positions. Theoretically, each - $\mathrm{OH}$ group found in a KDO molecule may contain a substitution. $\mathrm{KDO}^{21}$ and phosphate ${ }^{22}$ have been found attached to KDO at carbon position 4. Rietschel et al. ${ }^{22}$ have reported that carbon position 5 is substituted with such neutral sugars as L,D-heptose or D-mannose; that phosphoethanolamine can be present at the 7 carbon position of some bacterial KDO molecules; and that KDO or 4-amino-4-deoxy-L-arabinopyranose may be bound to the primary $-\mathrm{OH}$ in carbon position 8 of some KDO molecules. Strong acid hydrolysis enables the detection of KDO molecules substituted as described above, presumably by hydrolysis of the substituting group. ${ }^{16,21,23}$ We are currently trying to determine, by nuclear magnetic resonance, the nature and location of the substitutions on the KDO molecules that are only detectable by strong acid hydrolysis.

Finally, all $P$. cepacia LPS molecules that we tested were toxic for mice when injected i.p. We may conclude that substitutions do not influence toxicity of the LPS preparations of the various strains.

We thank David J. Hentges for his critical review of this manuscript. This study was supported by a Biomedical Research Grant from Texas Technical University Health Sciences Center and a grant from the Canadian Cystic Fibrosis Foundation to D.E.W. D.C.S. was supported by a Visiting Professor award from the Canadian Cystic Fibrosis Foundation. 


\section{REFERENCES}

1. Goldman DA, Klinger JD. Pseudomonas cepacia: biology, mechanisms of virulence and epidemiology. $J$ Pediatr 1986; 108: 806-812.

2. Corey M, Allison L, Prober C, Levison H. Sputum bacteriology in patients with cystic fibrosis in a Toronto hospital during 1979-1981. J Infect Dis 1984; 149: 283.

3. Isles A, MacLusky I, Corey M et al. Pseudomonas cepacia infection in cystic fibrosis: an emerging problem. $J$ Pediatr 1984; 104: 206-210.

4. Thomassen MJ, Demko CA, Klinger JD, Stern RC. Pseudomonas cepacia colonization among patients with cystic fibrosis. Am Rev Respir Dis 1985; 131 : 791-796.

5. Manniello JM, Heymann H, Adair FH. Isolation of atypical lipopolysaccharides from purified cell walls of Pseudomonas cepacia. J Gen Microbiol 1979; 112: 397-400.

6. Osborn MJ. Studies on the gram-negative cell wall. I. Evidence for the role of 2-keto-3-deoxyoctonate in the lipopolysaccharide of Salmonella typhimurium. Proc Natl Acad Sci USA 1963; 50: 499-506.

7. Anwar H, Brown MRW, Cozens RM, Lambert PA. Isolation and characterization of the outer and cytoplasmic membranes of Pseudomonas cepacia. J Gen Microbiol 1983; 129 : 499-507.

8. Straus DC, Lonon MK, Woods DE, Garner CW. Production of an extracellular toxic complex by various strains of Pseudomonas cepacia. J Med Microbiol 1989; 30: 17-22.

9. McKevitt AI, Retzer MD, Woods DE. Development and use of a serotyping scheme for Pseudomonas cepacia. Serodiagn Immunother 1987; 1 : 177-184.

10. Straus DC, Woods DE, Lonon MK, Garner CW. The importance of extracellular antigens in Pseudomonas cepacia infections. J Med Microbiol 1988; 26: 269-280.

11. Roberts RB, Abelson PH, Cowie DBJ, Bolton ET, Britten RJ. Studies of biosynthesis in Escherichia coli. Carnegie Institution of Washington Publication 607. Washington, DC. 1955.

12. Darveau RP, Hancock REW. Procedure for isolation of bacterial lipopolysaccharides from both smooth and rough Pseudomonas aeruginosa and Salmonella typhimurium strains. J Bacteriol 1983 ; 155: 831-838.

13. Lowry OH, Rosebrough NJ, Farr AL, Randall RJ. Protein measurement with the folin phenol reagent. J Biol Chem 1951 ; 193: 265-272.

14. Kabat EA, Mayer MM. Experimental immunochemistry, 2nd edn. Springfield, IL, Charles C Thomas. 1961 : 708.

15. Reed LJ, Muench H. A simple method of estimating fifty per cent endpoints. Am J Hyg 1938; 27 : 493-497.

16. Caroff M, Lebbar S, Szabó L. Do endotoxins devoid of 3deoxy-D-manno-2-octulosonic acid exist? Biochem Biophys Commun 1987; 143: 845-847.

17. Charon D, Szabó L. The synthesis of 3-deoxy-5-O-methyloctulosonic acid and its behaviour in the Warren reaction. Eur J Biochem 1972; 29: 184-187.

18. Hofstad T. The distribution of heptose and 2-keto-3deoxyoctonate in Bacteroidaceae. J Gen Microbiol 1974; 85: 314-320.

19. Wilkinson SG, Galbraith L, Lightfoot GA. Cell walls, lipids, and lipopolysaccharides of Pseudomonas species. Eur J Biochem 1973; 33: 158-174.

20. Howard SP, Buckley JT. Phospholipids and lipopolysaccharides of Aeromonas hydrophila. J Bacteriol 1985; 161 : $463-465$.

21. Brade H, Rietschel E-T. $\alpha$-2 $\rightarrow$ 4-Interlinked 3-deoxy-Dmanno-octulosonic acid disaccharide. A common constituent of enterobacterial lipopolysaccharide. Eur $J$ Biochem 1984; 145: 231-236.

22. Rietschel ET, Brade L, Schade U et al. Bacterial endotoxins: properties and structure of biologically active domains. In: E. Schrinner (ed) Surface structures of microorganisms and their interaction with the mammalian host (Workshop Conference Hoeschst vol 18). Weinheim, Verlag Chemie. 1988: 1-41.

23. Brade $\mathbf{H}, \mathbf{G a l a n o s} \mathrm{C}$, Luderitz $\mathrm{O}$. Differential determination of the 3-deoxy-D-manno-octulosonic acid residues in lipopolysaccharides of Salmonella minnesota rough mutants. Eur J Biochem 1983; 131 : 195-200. 